\title{
Atendimento da Estratégia Saúde da Família: percepções de usuários adultos e cuidadores de crianças
}

\section{Family Health Strategy Care: Adult users and children caregivers perceptions}

Maria Fernanda Pereira Gomes ${ }^{1}$, Kesley de Oliveira Reticena ${ }^{2}$, Lislaine Aparecida Fracolli ${ }^{3}$

1. ORCID: https://orcid.org/0000-0003-0577-2264. Doutora em Enfermagem. Universidade Paulista (UNIP), Assis, SP, Brasil. E-mail: m_fernanda_pgomes@ hotmail.com

2. ORCID: https://orcid.org/0000-0002-0905-588X. Mestre em Enfermagem. Universidade Paulista (UNIP), Assis, SP, Brasil. E-mail: kesleyreticena@ hotmail.com

3. ORCID: https://orcid.org/0000-0002-0936-4877. Doutora em Enfermagem. Escola de Enfermagem da Universidade de São Paulo (EEUSP), São Paulo, SP, Brasil. E-mail: lislaine@usp.br

CONTATO: Maria Fernanda Pereira Gomes | Universidade Paulista | Endereço: Rua Myrtes Spera Conceição, 301 - Conj. Nelson Marcondes, Assis-SP, CEP: 19813-550.

Telefone: (18) 3323-5500 - E-mail: m_fernanda_gomes@hotmail.com 
RESUMO O estudo buscou compreender a percepção de usuários da Atenção Primária à Saúde sobre o atendimento da Estratégia Saúde da Família na perspectiva de cuidadores de crianças menores de dois anos e usuários adultos. Trata-se de um estudo descritivo e qualitativo com a participação de 37 usuários adultos e 36 cuidadores de crianças, com média de idade de 38,2 anos, $89,04 \%$ mulheres e 10,96\% homens. As respostas passaram pela análise de conteúdo com a construção das temáticas: "Reconhecendo os atributos essenciais da Atenção Primária à Saúde", "Identificando fragilidades na Estratégia Saúde da Família" e "Sugerindo melhorias na Estratégia Saúde da Família". Os usuários da Estratégia Saúde da Família percebem a presença de atributos da Atenção Primária à Saúde como acesso de primeiro contato, integralidade e continuidade do cuidado, mas apresentam insatisfação com o agendamento de consultas para especialistas e exames diagnósticos, falta de medicamentos, falta de coordenação e integralidade na assistência à saúde.

DESCRITORES: Avaliação em Saúde. Atenção Primária a Saúde. Estratégia Saúde da Família. Coordenação do Cuidado. Rede de Atenção à Saúde.

ABSTRACT The study sought to understand the perception of Primary Health Care users regarding the fulfillment of the Family Health Strategy from the perspective of caregivers children of toddlers, infants and adult users.

This is a descriptive and qualitative study conducted with 37 adult users and 36 children caregivers, which presented a mean age of 38.2 years, with $89.04 \%$ of interviewees being female and $10.96 \%$ male. Answers underwent content analysis with the construction of the following themes: "Recognizing the essential attributes of Primary Health Care", "Identifying weaknesses in the Family Health Strategy" and "Suggesting improvements in the Family Health Strategy". Users of the Family Health Strategy perceive the presence of Primary Health Care attributes such as first contact access, integrality and care continuity, but are dissatisfied with scheduling appointments for specialists and diagnostic tests, lack of medication, lack of coordination and integrality in health care.

DESCRIPTORS: Health Evaluation. Primary Health Care. Family Health Strategy. Care Coordination. Health Care Network. 


\section{INTRODUÇÃO}

Atenção Primária à Saúde (APS) compreende um conjunto de ações em saúde formuladas a partir da Conferência Internacional sobre Cuidados Primários de Saúde em 1978. A Organização Mundial da Saúde (OMS), adotou os cuidados primários em saúde como meta para atingir o acesso de todas as pessoas a práticas de saúde contextualizadas à realidade local e cultural com a utilização de modos de atenção resolutivos, equitativos, integrais e a custos suportáveis ${ }^{1-2}$.

A Estratégia Saúde da Família (ESF), é o modelo assistencial brasileiro escolhido como prioritário para reorientação da APS. Inspira-se na declaração de Alma-Ata e busca fortalecer os princípios e diretrizes do Sistema Único de Saúde (SUS), possibilitando atendimento integral e contínuo às famílias e comunidades com base em seu espaço social, com atendimento de equipe multidisciplinar que trabalha na perspectiva da interdisciplinaridade, intervindo principalmente em situações de risco e vulnerabilidade social, priorizando a vigilância em saúde e a promoção da saúde².

No cenário atual a ESF descrita na Política Nacional de Atenção Básica (PNAB) 2017, não condiz com os pressupostos iniciais desse novo modelo assistencial, pois seu discurso é ambivalente sugerindo e recomendando que os gestores de saúde decidam sobre o número de agentes comunitários de saúde (ACS) por equipe, descaracterizando o diferencial da ESF de ampliação do acesso, cobertura de $100 \%$ da população e mapeamento de risco por meio de visita domiciliares ${ }^{3-4}$. A incorporação do profissional ACS nos territórios foi um grande diferencial que proporcionou uma maior vinculação entre a população e a equipe de saúde, além de orientar o trabalho com base nos determinantes do processo saúde-doença² .

A redação da PNAB 2012 é contrária a atual, pois estipula que para implantação e funcionamento de uma equipe de ESF, é necessário o número de ACS suficiente para cobrir $100 \%$ da população cadastrada. Cada ACS deve ser responsável por no máximo 750 pessoas ou 150 famílias, e o ideal é que cada equipe seja responsável por 3.000 pessoas com intuito de qualificar a assistência ${ }^{4-5}$. Nesta conjuntura, a APS brasileira que se alavancava com a expansão da ESF e seus modos de cuidar diferenciados, se encontra enfraquecida com a nova redação da PNAB.

Os serviços de APS no mundo se organizam em várias tipologias que apresentam características comuns como ser a porta de entrada principal do sistema de saúde, garantir a integralidade, coordenação e continuidade dos cuidados com foco na família e na comunidade ${ }^{1,6}$. Nesta perspectiva para resolução dos principais problemas de saúde que acometem a população, a APS deve coordenar a atenção à saúde de forma efetiva por meio de três vertentes: informação, clínica e organizacional ${ }^{7-9}$. A informação diz respeito à disponibilidade de informações sobre os usuários em todos níveis assistenciais e de 
fácil acesso para os profissionais ${ }^{8-9}$. A vertente clínica compreende uma APS assertiva e resolutiva que permite o cuidado sequencial e complementar entre os níveis de atenção ${ }^{8-9}$. A organizacional compreende os fluxos e a organização da rede de atenção à saúde que integra os níveis assistenciais de um sistema de saúde ${ }^{8-9}$.

Assim, uma das principais causas da falta de qualidade dos serviços de saúde de APS prestados é a ausência de coordenação do cuidado, que também se associa a custos elevados, duplicação e uso errôneo de procedimentos, diagnósticos, polifarmácia e plano terapêutico conflituoso ${ }^{10-11}$. Com intuito de fortalecer a coordenação da atenção, defendese a adequada operacionalização de Redes de Atenção à Saúde (RAS), com a intenção de superar a fragmentação da atenção e da gestão dos serviços de saúde ${ }^{1,6}$. A RAS é uma tecnologia de produção de ações e serviços de saúde que aproxima gestores de profissionais de saúde, de usuários e dos problemas de saúde reais da comunidade, permitindo a participação mais ativa desse ator na coordenação e gestão do cuidado, o que favorece positivamente a integralidade da atenção à saúde ${ }^{1,6}$.

Nesta perspectiva a presente pesquisa buscou compreender a percepção de usuários da APS sobre o atendimento de saúde disponibilizado pela ESF na perspectiva de cuidadores de crianças menores de dois anos e usuários adultos.

\section{MÉTODO}

Trata-se de um estudo descritivo, de natureza qualitativa, visto que esta oferece uma adequada apreensão da realidade de grupos particulares, além de propiciar a compreensão da subjetividade dos indivíduos ${ }^{12}$.

O estudo foi desenvolvido junto a usuários atendidos pelas ESF implantadas nos municípios que compõem as CIRs de Alto Capivari e Alta Sorocabana da RRAS 11 de Presidente Prudente, interior do Estado de São Paulo, Brasil.

As CIRs citadas são constituídas por uma totalidade de 24 municípios, sendo que a CIR Alta Sorocabana conta com 19 municípios: Alfredo Marcondes, Álvares Machado, Anhumas, Caiabu, Emilianópolis, Estrela do Norte, Indiana, Martinópolis, Narandiba, Pirapozinho, Presidente Bernardes, Presidente Prudente, Regente Feijó, Ribeirão dos Índios, Sandovalina, Santo Anastácio, Santo Expedito, Taciba e Tarabai; e a CIR Alto Capivari com cinco municípios: lepê, João Ramalho, Nantes, Quatá e Rancharia ${ }^{13}$.

Participaram do estudo 37 usuários adultos com idade igual ou superior a 18 anos e 36 cuidadores de crianças menores de dois anos, aplicando-se os seguintes critérios de inclusão: idade igual ou superior a 18 anos, ser atendido pela ESF há pelo menos um ano, condições físicas e cognitivas para responder aos questionamentos. 
Os dados foram coletados nos meses de junho a setembro de 2015 , sendo que a coleta foi realizada após o pesquisador abordar o usuário na unidade de saúde, a partir de seleção aleatória dentre os indivíduos que frequentavam as unidades no período da coleta.

Foram realizadas as seguintes questões: "Dê sua opinião sobre o atendimento de saúde à criança na ESF, aponte os pontos positivos e negativos deste tipo de serviço?" e "Dê sua opinião sobre o atendimento de saúde prestado aos adultos (maiores de 18 anos) na ESF, aponte os pontos positivos e negativos deste tipo de serviço?".

Os participantes foram entrevistados e as respostas transcritas à mão pelos pesquisadores em tempo real. Para a realização da análise dos dados, as respostas foram digitadas na íntegra no Microsoft Word for Windows e, após, passaram pelas três etapas do processo da análise de conteúdo na modalidade temática: a ordenação, a classificação e a análise final ${ }^{14}$. Deste processo emergiram as seguintes temáticas: "Reconhecendo os atributos essenciais da Atenção Primária à Saúde", "Identificando fragilidades na Estratégia Saúde da Família" e "Sugerindo melhorias na Estratégia Saúde da Família".

O desenvolvimento do estudo ocorreu em conformidade com o preconizado na resolução 466/2012 do Conselho Nacional de Saúde, tendo sido autorizado pelas CIRs e aprovado pelo Comitê de Ética em Pesquisa da Escola de Enfermagem da Universidade de São Paulo (CAAE: 42499615.9.0000.5392 e número de parecer: 1.024.138). Todos os participantes assinaram o termo de consentimento livre e esclarecido em duas vias de igual teor e, para garantir o anonimato dos mesmos, serão identificados como participante adulto e participante cuidador acompanhado do número na sequência em que participaram do estudo, acrescido com $M$ (referente ao município) também acompanhado do número na sequência em que participaram do estudo (Participante Adulto1M1, Participante Adulto2M2, Participante Adulto3M3...; Participante Cuidador1M1, Participante Cuidador2M2, Participante Cuidador3M3...).

\section{RESULTADOS}

Participaram do estudo 37 usuários adultos e 36 cuidadores de crianças menores de dois anos, com média de idade de 38,2 anos, $89,04 \%(n=65)$ sexo feminino e $10,96 \%$ $(\mathrm{n}=8)$ sexo masculino.

Tanto os usuários adultos quanto os cuidadores de crianças menores de dois anos apontaram pontos positivos e negativos quanto ao atendimento da ESF em seus respectivos municípios, sendo que as opiniões convergiram em três temáticas que serão apresentadas a seguir. 


\section{Reconhecendo os atributos essenciais da Atenção Primária à Saúde}

Ao analisar as respostas dos participantes, observou-se que os participantes adultos e os cuidadores de crianças menores de dois anos reconheceram a qualidade da assistência prestada pela ESF como satisfatória. Os participantes adultos evidenciaram que foram atendidos em tudo o que precisaram e que o cuidado é realizado integralmente:

Na minha opinião o atendimento está ótimo, pois minhas necessidades são atendidas. (Participante Adulto1M1)

O atendimento na Unidade é muito bom, os profissionais mostramse interessados em ajudar em todas as demandas possíveis, mesmo quando não é dá área da saúde. Estão sempre prontos a ajudar no que for necessário. (Participante Adulto7M5)

Evidencia-se, além da integralidade, a presença de outros atributos essenciais da APS, como a longitudinalidade:

Os profissionais da ESF são muito próximos da população, participativos ativos da vida dos usuários, acolhedores e sempre prontos para resolver todas as demandas trazidas. Quando é necessário encaminhamento, a equipe que agenda e acompanha do começo ao fim o processo. Não tem pontos negativos a respeito do serviço prestado pela ESF. (Participante Cuidador32M18)

Os cuidadores de crianças menores de dois anos também evidenciam o cuidado humanizado e integral, o acesso de primeiro contato na unidade e a organização da unidade de saúde:

Todas as situações que precisou do serviço da ESF tanto para a mãe como para os filhos, seja por rotina ou situação emergencial de saúde, foi bem atendida e seu caso solucionado sem demorar [...]. (Participante Cuidador8M5)

O atendimento é muito bom na unidade, o médico muito atencioso, investiga o problema com profundidade, solicita todos os exames para detectar o diagnóstico preciso para melhor tratar a queixa. Não precisou procurar um médico particular, pois está muito satisfeita. (Participante Cuidador15M10)

Os funcionários da ESF são prestativos para a resolução da queixa dos pais em relação à criança; a unidade está sempre limpa e organizada, proporcionando um clima agradável [...]. (Participante Cuidador27M15)

Os relatos mostram que os profissionais que trabalham na ESF propiciam o acesso, a continuidade do cuidado tanto aos usuários adultos quanto às crianças, bem como atenção integral, realizando as referências necessárias. 


\section{Identificando fragilidades na Estratégia Saúde da Família}

Observou-se que, apesar de alguns usuários reconhecerem a qualidade da assistência prestada pelas equipes ESF e a presença dos atributos essenciais da APS, foi grande 0 número de participantes que apontou pontos frágeis dos serviços, sendo que dentre os usuários adultos, alguns relataram demora no atendimento, em conseguir consultas com especialistas e realizar exames diagnósticos:

Sou bem atendido pela equipe do posto, mas quando precisa de médico especialista o agendamento demora muito. (Participante Adulto2M2)

[...] Ponto negativo: demora de exames como ultrassom. Só quando marca com urgência que é mais rápido. (Participante Adulto14M10)

$O$ atendimento e funcionários são muitos bons, mas quando precisa de dentista e especialista não tem, quando marca demora para atender quando não se perde o encaminhamento e a gente nem fica sabendo, tem que vir atrás e remarcar novamente. (Participante Adulto19M13)

A mesma fragilidade esteve presente nos relatos dos participantes cuidadores:

[...] Pontos negativos a demora para consultar sempre tem muita gente. A demora para alguns exames e para outros médicos. (Participante Cuidador11M7)

[...] De ruim, só a demora para consultar e para fazer exames. (Participante Cuidador13M9)

[...] Pontos negativos: Demora de exames como o vascular, oftalmo., otorrino etc. (Participante Cuidador16M10)

Além dessas fragilidades, também foi citada a falta de profissionais e medicações por adultos e cuidadores:

[...] Pontos negativos: ter mais acesso a medicação, pois falta remédios no posto. (Participante Adulto22M14)

[...] De ruim tem muita gente e só tem um médico. (Participante Adulto36M20)

Os cuidadores evidenciaram ainda, a falta de médico pediatra:

[...]Só acho que deveria ter mais que um médico para os atendimentos ou um pediatra só para as crianças. (Participante Cuidador3M2)

Todas as vezes que precisei de consulta para minha criança fui bem atendida, mas precisava ter na minha cidade um Pediatra para melhorar mais. (Participante Cuidador5M3)

[...] Único ponto ruim é o fato de não ter um pediatra no posto, apenas clínico geral. (Participante Cuidador29M16) 
Os relatos sugerem que, apesar de serem bem atendidos, alguns pontos de atenção dos serviços necessitam de investimentos para que seja alcançada a integralidade e qualidade da assistência.

\section{Sugerindo melhorias na Estratégia Saúde da Família}

Além das principais fragilidades da APS apontadas pelos participantes (demora e falta de profissionais), outros pontos, vistos como desafios do serviço e dos profissionais apareceram nos relatos. Dentre os participantes adultos, alguns ressaltaram questões relativas ao horário de funcionamento da unidade e à qualidade da assistência, o que dificulta o atendimento aos atributos essenciais da APS:

[...] após as 17 horas tem que ser encaminhado para a Santa Casa, se precisar de atendimento, acho que deveria ter um pronto atendimento mais próximo ou ficar aberto até mais tarde. (Participante Adulto4M2)

Pontos negativos: a recepção (funcionários) necessita realizar atendimento humanizado. A equipe da UBS necessita trabalhar mais a ética profissional[...]. (Participante Adulto8M6)

[...]Ponto negativo: fico surpreso muitas vezes com ofato de algumas enfermeiras não serem compreensivas com o desespero e alteração do humor de pessoas que precisam de um atendimento mais rápido[...]. (Participante Adulto21M14)

[...] Negativos: gostaria que tivesse mais horários para as atividades grupais, pois, devido ao trabalho, não consegue participar. (Participante Adulto28M15)

Além destes desafios, cuidadores de crianças menores de dois anos também ressaltaram a qualidade da assistência, a rotatividade de profissionais, a falta de informação dos serviços que são prestados, a limitação na composição da equipe de saúde e a desorganização nas unidades.

O atendimento na unidade é muito básico. Os profissionais não investigam com profundidade o quadro clínico, deixando de prestar uma assistência integral à saúde. A rotatividade de médicos é muito grande, prejudicando a continuidade do cuidado. (Participante Cuidador7M4)

[...] O negativo é a falta de informação dos serviços que são prestados. (Participante Cuidador10M7)

A equipe de saúde é muito boa, acolhedora, buscando sempre ajudar no que for preciso, porém é limitada em termos de multidisciplinaridade, dificultando uma assistência integral ao usuário, até em situações rotineiras. (Participante Cuidador33M19)

[...] Os profissionais não têm muito envolvimento com o que se passa de fato com o usuário, perdem prontuários devido desorganização, porém respeitam os usuários e tentam encaminhar para os serviços necessários quando não tem no posto. (Participante Cuidador36M21) 
Observa-se, a partir dos relatos, que existem diversas questões específicas a respeito da qualidade da assistência e ao funcionamento dos serviços que merecem mais atenção para que os princípios do sistema de saúde brasileiro sejam operacionalizados.

\section{DISCUSSÃo}

Ficou evidente ao analisar a percepção dos usuários da ESF da Região de Presidente Prudente que os participantes percebem a presença de atributos da APS como acesso de primeiro contato, integralidade e continuidade do cuidado. Observou-se também que os usuários apresentam insatisfação com o agendamento de consultas para especialistas e exames diagnósticos, falta de medicamentos, falta de coordenação e integralidade na assistência à saúde. Outros problemas relatados são a falta de comprometimento e falta de organização da equipe de saúde, demora para ser atendido na unidade de saúde, falta de médico pediatra para acompanhar as crianças, rotatividade de médicos e pouca informação sobre o funcionamento dos serviços. Destaca-se também que os usuários sugerem mudanças no atendimento da ESF como horários estendidos para o atendimento à população e aumentar o número de médicos.

Resultados semelhantes foram obtidos na pesquisa realizada em dois municípios do Estado do Pernambuco, no qual os usuários da ESF referiram dificuldades para ter acesso a exames, consultas com especialistas e urgências como a demora para o agendamento e para ser atendido ${ }^{15}$. De acordo com outra pesquisa, as razões associadas a não-resolubilidade do sistema são a falta de remédios, poucas vagas e demora para o atendimento, dificuldade no sistema de referência e contrarreferência e poucos profissionais em atividade ${ }^{16}$. Neste caso, referência é considerada o ato de encaminhar o usuário de um serviço para outro de maior complexidade, enquanto a contrarreferência é o ato de devolver o usuário para o serviço que o encaminhou com informações detalhadas sobre a situação de saúde ${ }^{17-18}$. Ademais, o funcionamento sincrônico do sistema de referência e contrarreferência é o ponto chave para a viabilização de um sistema coordenado ${ }^{18}$.

A realidade encontrada na presente pesquisa corrobora com a situação de vários municípios brasileiros que enfrentam problemas na operacionalização da APS como porta de entrada preferencial do sistema, horários restritos de funcionamento, dificuldade de manejo da demanda espontânea e baixa resolutividade ${ }^{19}$.

Nesta perspectiva, observa-se na presente pesquisa que a coordenação da atenção à saúde é fragmentada, apresentando barreiras de acesso aos serviços especializados, ambulatoriais e hospitalares, filas por serviços de saúde, falta de comunicação entre a APS e os serviços especializados, gestão de recursos inadequada como exames complementares duplicados e referências desnecessárias, além da utilização inadequada 
dos serviços de urgência e emergência ${ }^{20-21}$. As barreiras organizacionais persistem no acesso, nos fluxos pouco ordenados e na integração da APS à rede ${ }^{22}$.

Os serviços especializados devem ser acessíveis e agendados em tempo, adequado às necessidades de saúde dos usuários e aos riscos e urgência da situação de saúde por eles apresentada ${ }^{1}$. As dificuldades de acesso à APS aumentam a demanda por atenção não urgente nos serviços de urgência e emergência, o que mostra que a falta de acesso a um ponto de atenção reflete negativamente em outros serviços do sistema de saúde ${ }^{21,23}$.

No que tange a integralidade dos serviços de saúde disponíveis e prestados na Região de Presidente Prudente, os usuários referem um descompasso na gestão dos fluxos de atendimento para outros níveis assistenciais, especialidades e exames diagnósticos. Relatam longo período de espera para ter acesso e que falta profissionais na rede de serviços, neste quadro para garantir a integralidade. A ESF precisa tornar-se a porta de entrada do sistema e integrar-se à rede assistencial assumindo o papel de coordenadora do cuidado dos usuários ${ }^{24-25}$. A insuficiência de serviços especializados de referência pode estar relacionada à uma expansão da APS por meio da ESF, sem a simultânea expansão e desenvolvimento da atenção especializada ${ }^{24,26}$.

Ademais, as dificuldades de acesso aos serviços de saúde são potencializadas pela fragmentação da atenção à saúde das organizações públicas e privadas que não trabalham com a integração de todos os serviços em redes de atenção à saúde o que torna o sistema de saúde menos resolutivo, ineficiente e de pouca qualidade ${ }^{21}$. Neste contexto, uma forma para melhorar os problemas de acesso seria o fortalecimento e qualificação da APS, que deve resolver a maioria dos problemas de saúde, coordenar os fluxos de atendimentos das pessoas a outros níveis assistenciais e coordenar as informações ao longo de toda a rede de serviços ${ }^{21}$.

No Brasil, a Rede de Atenção à Saúde (RAS) é uma organização do conjunto de serviços de saúde, não hierárquicos, ligados por ações cooperativas que pretendem garantir atenção contínua e integral a população sob sua responsabilidade ${ }^{9,27}$. As características de uma RAS funcional e dinâmica que tem a APS como porta de entrada e coordenadora, está associada a serviços de saúde de qualidade, custo-efetivos, que trazem satisfação aos usuários e melhores resultados globais de saúde ${ }^{9,28}$.

Na ESF da Região de Presidente Prudente evidencia-se que no âmbito de sua governabilidade local apresenta esforços para atendimento à população de qualidade e de forma integral, mas não consegue ser resolutiva quando os usuários possuem necessidades que abrangem outros níveis assistenciais. Neste contexto, é primordial o papel da APS na coordenação do cuidado, porém é importante refletir sobre as reais causas do problema, como falta de especialistas, rede de saúde desestruturada que 
impede a movimentação do usuário, falta de informação, ineficiência do sistema de referência e contrarreferência, utilização errônea de recursos da saúde como a solicitação de avaliação de especialistas e de exames diagnósticos de forma indiscriminada.

Nesta perspectiva a avaliação da satisfação dos usuários dos serviços de saúde é imprescindível para a construção de estratégias e decisões mais assertivas com intuito de rever as práticas profissionais, o processo de trabalho, distribuição e utilização de recursos, a organização dos serviços em rede, a resolutividade dos serviços disponíveis e a readequação e formulação de políticas ${ }^{29}$.

\section{CONSIDERAÇÕES FINAIS}

A ESF na Região de Presidente Prudente de acordo com a percepção dos usuários adultos e cuidadores de crianças menores de dois anos, possui pontos positivos como a humanização e longitudinalidade do cuidado e pontos negativos como demora para agendar e ser atendido por especialistas e realizar exames diagnósticos, falta de medicamentos, falta de coordenação e integralidade do cuidado. Nesta perspectiva, a Coordenação do Cuidado e a Integralidade se mostram frágeis na Região, fato que pode ser melhorado com a eficiente operacionalização do Sistema de Referência e Contrarreferência e Redes de Atenção à Saúde. Neste contexto, sugere-se que novas pesquisas sejam realizadas com a finalidade de elucidar as questões levantadas no presente estudo.

\section{REFERÊNCIAS}

1. Starfield $B$, organizadora. Atenção Primária: equilíbrio entre necessidades de saúde, serviços e tecnologia. Brasília: Organização das Nações Unidas para a Educação, a Ciência e a Cultura; 2002.

2. Fertonani HP, Pires DEP, Biff D, Scherer MDA. The health care model: concepts and challenges for primary health care in Brazil. Ciênc. saúde coletiva [Internet]. 2015 [citado em 2017 Mar 12];20(6):18691878. Disponível em: https://doi.org/10.1590/1413-81232015206.13272014.

3. Brasil. Ministério da Saúde. Portaria n. 2.436, de 21 de setembro de 2017. Aprova a Política Nacional de Atenção Básica, estabelecendo a revisão de diretrizes para a organização da Atenção Básica, no âmbito do Sistema Único de Saúde (SUS). Diário Oficial da União, Brasília, p. 68, 22 set. 2017. Seção 1.

4. Morosini MVGC, Fonseca AF, Lima LD. Política Nacional de Atenção Básica 2017: retrocessos e riscos para o Sistema Único de Saúde. Saúde debate [Internet]. 2018 [citado em 2018 Abr 10];42(116):11-24. Disponível em: https://doi.org/10.1590/0103-1104201811601.

5. Ministério da Saúde (BR). Secretaria de Atenção à Saúde. Departamento de Atenção Básica. Política Nacional de Atenção Básica [Internet]. Brasília: Ministério da Saúde; 2012 [citado em 2017 Abr 10]. Disponível em: http://189.28.128.100/dab/docs/publicacoes/geral/pnab.pdf.

6. Lapão LV, Arcêncio RA, Popolin MP, Rodrigues LBB. The role of Primary Healthcare in the coordination of Health Care Networks in Rio de Janeiro, Brazil, and Lisbon region, Portugal. Ciênc. saúde coletiva [Internet]. 2017 [citado em 2017 Jun 12];22(3):713-724. Disponível em: https://doi.org/10.1590/141381232017223.33532016. 
7. Mendes, EV. A construção social da atenção primária à saúde. / Eugênio Vilaça Mendes. Brasília: Conselho Nacional de Secretários de Saúde - CONASS; 2015. 193 p.

8.Vargas I, Mogollon-Perez AS, De Paepe P, Ferreira da Silva MR, Unger JP, Vazquez ML. Barriers to healthcare coordination in market-based and decentralized public health systems: a qualitative study in healthcare networks of Colombia and Brazil. Health Policy Plan [Internet]. 2016 [citado em $2017 \mathrm{Abr}$ 23];31(6):736-748. Disponível em: https://pubmed.ncbi.nlm.nih.gov/26874327/.

9. Bousquat A, Giovanella L, Campos EMS, Almeida PF, Martins CL, Mota PHS et al. Primary health care and the coordination of care in health regions: managers' and users' perspective. Ciênc. saúde coletiva [Internet]. 2017 [citado em 2017 Set 23];22(4):1141-1154. Disponível em: https://doi.org/10.1590/141381232017224.28632016.

10. Bynum JPW, Ross JS. A measure of care coordination? J Gen Intern Med [Internet]. 2012 [citado em 2017 Abr 23];28(3):336-8. Disponível em: https://www.ncbi.nlm.nih.gov/pmc/articles/PMC3579982/.

11. Almeida PF, Santos AM. Primary Health Care: care coordinator in regionalized networks? Rev Saúde Pública [Internet]. 2016 [citado em 2017 Abr 23];50(80):1-13. Disponível em: https://doi.org/10.1590/s15188787.2016050006602.

12. Gomes R. A análise de dados em pesquisa qualitativa. In: Minayo, MCS Pesquisa social: teoria, métodos e criatividade. $20^{\mathrm{a}}$ ed. Rio de Janeiro: Vozes; 2010.

13. Sala de Apoio a Gestão Estratégica (SAGE) do Ministério da Saúde [homepage na internet]. Brasília: Ministério da Saúde; 2011. [citado 2019 dez. 9]. Disponível em: http://sage.saude.gov.br/.

14. Minayo MCS. O desafio do conhecimento: pesquisa qualitativa em saúde. 12. ed. São Paulo (SP): Hucitec; 2010.

15. Samico I, Hartz ZMA, Felisberto E, Carvalho EF. Atenção à saúde da criança: uma análise do grau de implantação e da satisfação de profissionais e usuários em dois municípios do estado de Pernambuco, Brasil. Rev. Bras. Saude Mater. Infant. [Internet] 2005 [citado em 2017 Dez 21]; 5(2):229-240. Disponível em: https://doi.org/10.1590/S1519-38292005000200012.

16. Moimaz SAS, Marques JAM, Saliba O, Garbin CAS, Zina LG, Saliba NA. Satisfação e percepção do usuário do SUS sobre o serviço público de saúde. Physis [Internet]. 2010 [citado em 2017 Dez 21];20(4):1419-1440. Disponível em: https://doi.org/10.1590/S0103-73312010000400019.

17. Melo DF, Criscuolo MBR, Viegas SMF. Reference and counter-reference in everyday health care in Minas Gerais, Brazil: the support to decisions of primary care. Rev Fund Care Online [Internet]. 2016 [citado em 2017 Dez 21];8(4):4986-4995. Disponível em: http://dx.doi.org/10.9789/2175-5361.rpcfo.v8.4402.

18. Fernandes LA, Neves RS, Leandro SS, Monteiro PS. Coordenação do cuidado em uma região administrativa do distrito federal: uma pesquisa avaliativa. Enferm. Foco [Internet]. 2018 [citado em 2019 Jan 13];9(1):13-19. Disponível em: http://revista.cofen.gov.br/index.php/enfermagem/article/view/1554/420

19. Almeida PF, Fausto MCR, Giovanella L. Fortalecimento da atenção primária à saúde: estratégia para potencializar a coordenação dos cuidados. Rev Panam Salud Publica [Internet]. 2011 [citado em 2017 Dez 21];29(2):84-95. Disponível em: https://scielosp.org/article/rpsp/2011.v29n2/84-95/.

20. Vargas I, Vázquez ML, Mogollón-Pérez AS, Unger JP. Barriers of access to care in a managed competition model: lessons from Colombia. BMC Health Serv Res. [Internet]. 2010 [citado em 2017 Dez 21]; 10:297. Disponível em: https://www.ncbi.nlm.nih.gov/pmc/articles/PMC2984497/.

21. Mendes EV. O acesso à atenção primária à saúde. Brasília: Conselho Nacional de Secretários de Saúde - CONASS; 2016. 171 p.

22. Fausto MCR, Giovanella L, Mendonça MHM, Seidl H, Gagno J. A posição da Estratégia Saúde da Família na rede de atenção à saúde na perspectiva das equipes e usuários participantes do PMAQAB. Saúde debate [Internet]. 2014 [citado em 2017 Dez 21];38(n. spe):13-33. Disponível em: https://doi. org/10.5935/0103-1104.2014S003.

23. Brousseau DC, Bergholte J, Gorelick MH. The effect of prior interactions with a primary care provider on nonurgent pediatric emergency department use. Arch Pediatr Adolesc Med. [Internet]. 2004 [citado em 2017 Dez 21];158(1):78-82. Disponível em: https://jamanetwork.com/journals/jamapediatrics/fullarticle/485603.

24. Arantes LJ, Shimizu HE, Merchán-Hamann E. The benefits and challenges of the Family Health Strategy in Brazilian Primary Health care: a literature review. Ciênc. saúde coletiva. [Internet]. 2016 [citado em 2017 
Dez 21];21(5):1499-1509. Disponível em: https://www.scielo.br/scielo.php?script=sci_arttext\&pid=S1413$81232016000501499 \&$ Ing $=$ en\&nrm=iso\&tIng=pt\&ORIGINALLANG=pt.

25. Nascimento DDG, Oliveira MAC. Reflexões sobre as competências profissionais para o processo de trabalho nos núcleos de apoio à saúde da família. Mundo Saude [Internet]. 2010 [citado em 2017 Dez 21]; 34(1):92-96. Disponível em: http://www.saocamilo-sp.br/pdf/mundo_saude/74/12_revisao_reflexoes.pdf.

26. Os autores respondem. Quinze anos de uma agenda em construção: diálogos sobre o PSF no Brasil. Ciênc. saúde coletiva [Internet]. 2009 [citado em 2017 Dez 21]; 14(Suppl 1): 1343-1345. Disponível em: https://doi.org/10.1590/S1413-81232009000800006.

27. Ministério da Saúde (BR). Portaria GM/MS 4.279, de 30 de dezembro de 2010. Estabelece diretrizes para a organização da Rede de Atenção à Saúde no âmbito do Sistema Único de Saúde (SUS). Diário Oficial da União, Brasília, 31 dez. 2010.

28. Kringos DS, Boerma WG, Bourgueil Y, Cartier T, Hasvold T, Hutchinson A et al. The European primary care monitor: structure, process and outcome indicators. BMC Fam Pract. [Internet]. 2010 [citado em 2017 Dez 21];11:81. Disponível em: https://bmcfampract.biomedcentral.com/articles/10.1186/1471-2296-11-81.

29. Brandão ALRBS, Giovanella L, Campos CEA. Avaliação da atenção básica pela perspectiva dos usuários: adaptação do instrumento EUROPEP para grandes centros urbanos brasileiros. Ciênc. saúde coletiva [Internet]. 2013 [citado em 2017 Dez 21];18(1):103-114. Disponível em: https://www.scielo.br/ scielo.php?script=sci_arttext\&pid=S1413-81232013000100012\&lng=en\&nrm=iso\&tlng=pt.

RECEBIDO: 11/07/2020

ACEITO: 07/10/2020 